\title{
Charmonium radiative transitions, meson and glueball particle properties with the effective strong coupling
}

\author{
Gurjav Ganbold ${ }^{1,2, *}$ \\ ${ }^{1}$ Bogoliubov Laboratory of Theoretical Physics, JINR, Dubna 141980, Russia \\ ${ }^{2}$ Institute of Physics and Technology, Ulaanbaatar 13330, Mongolia
}

\begin{abstract}
The particle properties of conventional mesons and scalar glueball, radiative transitions of charmonium excited states $\chi_{c J}(J=0,1,2)$ are studied in the framework of relativistic quark models with infrared confinement by taking into account the mass dependence of the effective strong coupling. A specific behaviour of the mass-dependent strong coupling with a freezing point $\alpha_{s}(0)=$ 1.032 has been revealed. The spectrum and leptonic (weak) decay constants of conventional mesons have been calculated in good agreement with the latest experimental data. New estimates on the scalar glueball mass, 'radius' and gluon condensate value have been obtained. Dominant radiative transitions of the charmonium orbital excitations $\chi_{c J} \rightarrow J / \Psi+\gamma$ have been studied and the partial decay widths have been estimated with reasonable accuracy.
\end{abstract}

\section{Introduction}

In modern particle physics one deals with a number of phenomena such as the quark confinement, running strong coupling, generation of hadron mass etc., which require correct description of hadron dynamics in the low-energy domain within theoretical models. QCD predicts a dependence of the physical strong coupling under changes of energy (or, mass) scale $Q$. This dependence $\alpha_{s}(Q) \doteq g^{2} /(4 \pi)$ is determined well in experiments at relatively high energies [1]. Meanwhile, the low-energy (or, infrared - IR) behavior of $\alpha_{s}$ has not been well defined yet, it needs to be more specified, because many quantities in particle physics are affected by the IR behavior of $\alpha_{s}$. The correct description of QCD effective coupling in the IR regime remains one of the important problems. The hadron mass origin is one of the challenges to particle physics because the Standard Model introduces only fundamental particles and does not explain the appearance of the multitude of observed massive hadrons. The calculation of hadron mass spectra qualitatively comparable to the precise experimental data, still remains a key problem. Recently, the Particle Data Group [2] has reported that the treatment of the branching ratios of the charmonium excited states $\chi_{c J}(J=0,1,2)$ have undergone an important restructuring. The study of the properties of $\chi_{c J}$ mesons is of great interest due to some of their outstanding features.

Our study is based on the formalism of analytic (infrared) confinement principle [3] and the Covariant Confined Quark Model (CCQM) [4].

\footnotetext{
*e-mail: ganbold@theor.jinr.ru
} 


\section{Model with infrared confinement}

One of the theoretical approaches explaining the non-observation of quarks is the Analytic Confinement model assuming entire analytic nonlocal functions for the quark and gluon propagators [3]. The QCD-inspired model Lagrangian reads [5]:

$$
\mathcal{L}=-\frac{1}{4}\left(\partial^{\mu} \mathcal{A}_{v}^{A}-\partial^{v} \mathcal{A}_{\mu}^{A}+g f^{A B C} \mathcal{A}_{\mu}^{B} \mathcal{A}_{v}^{C}\right)^{2}+\left(\bar{q}_{f}^{a}\left[\gamma_{\alpha} \partial^{\alpha}-m_{f}\right] q_{f}^{b}\right)+g\left(\bar{q}_{f}^{a}\left[\Gamma_{C}^{\alpha} \mathcal{A}_{\alpha}^{C}\right] q_{f}^{b}\right),
$$

where $\mathcal{A}_{\alpha}^{C}$ is the gluon and $q_{f}^{a}$ is a quark field of flavor $f$ with mass $m_{f}=\left\{m_{u d}, m_{s}, m_{c}, m_{b}\right\}$ and $\Gamma_{C}^{\alpha}=i \gamma_{\alpha} t^{C}$. For the spectra of quark-antiquark and di-gluon bound states we solve BetheSalpeter type equations in [6]. The master equation for the meson mass reads [5]:

$$
\begin{aligned}
1=\alpha_{s} \cdot \lambda_{J J}\left(M_{J}^{2}, m_{1}, m_{2}\right)= & \alpha_{s} \frac{16 \pi C_{J}}{9} \int \frac{d^{4} k}{(2 \pi)^{4}} \iint d x d y e^{-i k(x-y)} U_{\mathcal{N}}(x) \sqrt{D(x) D(y)} \\
& \times\left. U_{\mathcal{N}}(y) \operatorname{Tr}\left[O_{J} \tilde{S}_{m_{1}}\left(\hat{k}+\xi_{1} \hat{p}\right) O_{J} \tilde{S}_{m_{2}}\left(\hat{k}-\xi_{2} \hat{p}\right)\right]\right|_{-p^{2}=M_{J}^{2}},
\end{aligned}
$$

where $C_{J}=\{1,1,1 / 2,-1 / 2\}, \xi_{i}=m_{i} /\left(m_{1}+m_{2}\right), O_{J}=\left\{I, i \gamma_{5}, i \gamma_{\mu}, \gamma_{5} \gamma_{\mu}\right\}$ and the gluon $(\tilde{D}(k))$ and quark propagator $\left(\tilde{S}_{m_{1}}(\hat{p})\right)$ are represented in Euclidean momentum space.

Note, the polarization kernel $\lambda_{J J^{\prime}}\left(-p^{2}\right)$ has to be diagonalized using a complete system of orthonormal functions $\left\{U_{\mathcal{N}}\right\}$, where $\mathcal{N}=\{n, l, \mu, \ldots\}$ is a set of quantum numbers. The solution of Eq. (2) is nothing else but the solution of the corresponding ladder Bethe-Salpeter equation.

Ultraviolet divergences in the model have been removed by renormalization of wave function and charge, but infrared (IR) singularities remain because of integration over momentum $k$ in Eq. (2). To avoid the appearance of the singularities in the mass formula, we follow theoretical predictions in favor of an IR-finite behavior of the gluon propagator [7] and introduce IR cutoffs on the limits of scale integrations for the propagators as follows:

$$
D(x)=\frac{1}{4 \pi^{2} x^{2}} \rightarrow \frac{1}{4 \pi^{2}} \int_{\Lambda^{2} / 2}^{\infty} d s e^{-s x^{2}}, \quad \tilde{S}_{m_{f}}(\hat{p}) \rightarrow\left(i \hat{p}+m_{f}\right) \int_{0}^{1 / \Lambda^{2}} d t e^{-t\left(p^{2}+m_{f}^{2}\right)},
$$

where $\Lambda$ is the mass scale parameter characterizing the IR confinement domain. These propagators are entire analytic functions in the Euclidean space for $\Lambda>0$. Note, another type of IR confinement applied to whole 'quark-antiquark' loop in [4] that is used in Sect. 3.

\section{Effective strong coupling in the IR region}

The meson mass $M_{J}$ is defined by Eq. (2) at given $\alpha_{s}$, quantum numbers $\mathcal{N}$ and constituent quark masses $\left\{m_{1}, m_{2}\right\}$. And vice versa, $\alpha_{s}$ can be estimated for given mass. The QCD

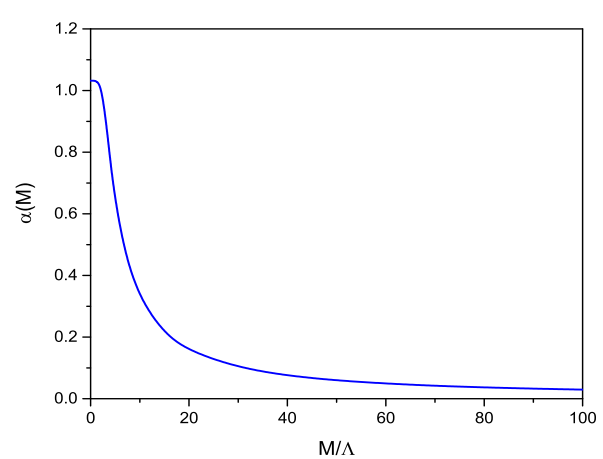

coupling may feature an IR-finite behavior (e.g., in [8]). To study this, we consider $M_{J}$ as
Figure 1. Effective strong coupling $\alpha_{s}$ in dependence on the relative mass scale $M / \Lambda$. The slope of $\alpha_{s}(M / \Lambda)$ changes for different $\Lambda>0$, but the origin $\alpha_{s}(0)=1.032$ remains fixed 
an appropriate energy-scale for $\alpha_{s}$ and choose a specific case $m_{1}=m_{2} \sim M / 2$. Then, a new effective (mass-dependent) strong coupling $\alpha_{s}(M)$ in time-like domain reads:

$$
\alpha_{s}(M)=-1 / \lambda(M, M / 2, M / 2) .
$$

The dependence of the effective strong coupling $\alpha_{s}(M / \Lambda)$ on a relative mass scale $M / \Lambda$ is shown in Fig. 1. Generally, the slope of $\alpha_{s}(M / \Lambda)$ changes for different $\Lambda>0$, but the newly revealed origin $\alpha_{s}^{0}=\alpha_{s}(0)=1.032$ remains unchanged. This upper bound to the IR-fixed value $\alpha_{s}^{0} / \pi=0.328$ is in a reasonable agreement with often quoted estimate in [9].

\section{Lowest glueball state}

The existence of glueballs, the bound states of gluons, is predicted by QCD because of the self-interaction of gluons. There are predictions expecting glueball-like states in the mass range $M_{G} \sim 1.5 \div 5.0 \mathrm{GeV}$ with spin $J=0,1,2,3$ [2]. Below we consider a two-gluon scalar bound state with $J^{P C}=0^{++}$and define the scalar glueball mass $M_{0^{++}}$from the equation:

$$
1-\frac{8 \alpha_{s}\left(M_{0^{++}}\right)}{3 \pi} \int d z e^{i z p} \Pi_{G}(z)=0, \quad p^{2}=-M_{0^{++}}^{2},
$$

where $\Pi_{G}(z)$ is the self-energy (polarization) function of the scalar glueball.

Particularly, for $\Lambda=236 \mathrm{MeV}$ and $\alpha\left(M_{G}\right)$ defined from Eq. (4) we obtain new estimates:

$$
M_{0^{++}}=1739 \mathrm{MeV}, \quad \alpha_{s}\left(M_{0^{++}}\right)=0.451 .
$$

The new value of $M_{0^{++}}$is in reasonable agreement with other predictions [2, 6, 10-12].

We also have obtained the scalar glueball 'radius' $\left(r_{0^{++}}\right)$that valued a often referred combination as follows: $r_{0^{++}} \cdot M_{0^{++}}=4.41$ in reasonable agreement with data in [2].

\section{Meson spectrum}

The dependence of meson mass on $\alpha_{s}$ and other model parameters is defined by Eq. (2). The kernel function $\lambda_{\mathcal{N}}$ is real and finite, that allows us to evaluate a variational solution to $M_{J}$. For the fixed above parameter $\Lambda=236 \mathrm{MeV}$ and the $\alpha_{s}(M)$ defined in Eq. (4) we derive meson mass formula Eq. (2) by fitting the conventional meson masses with adjustable constituent quark masses $\left\{m_{u d}, m_{s}, m_{c}, m_{b}\right\}$. We have fixed the final set of model parameters (in units of $\mathrm{MeV}$ ) as follows:

$$
\Lambda=236, m_{u d}=227.6, m_{s}=420.1, m_{c}=1521.6, m_{b}=4757.2
$$

and represent in Tab. 1 our new estimates on the pseudoscalar $(P)$ and vector $(V)$ meson masses.

Table 1. Estimated masses of conventional mesons compared to the experimental data

\begin{tabular}{llllll}
\hline$J^{P C}=0^{-+}$ & $M_{P}(\mathrm{MeV})$ & Data $[2]$ & $J^{P C}=1^{--}$ & $M_{V}(\mathrm{MeV})$ & Data \\
\hline$D$ & 1893.6 & 1869.62 & $\rho$ & 774.3 & 775.26 \\
$D_{s}$ & 2003.7 & 1968.50 & $K^{*}$ & 892.9 & 891.66 \\
$\eta_{c}$ & 3032.5 & 2983.70 & $D^{*}$ & 2003.8 & 2010.29 \\
$B$ & 5215.2 & 5259.26 & $D_{s}^{*}$ & 2084.1 & 2112.3 \\
$B_{s}$ & 5323.6 & 5366.77 & $J / \Psi$ & 3077.6 & 3096.92 \\
$B_{c}$ & 6297.0 & 6274.5 & $B^{*}$ & 5261.5 & 5325.2 \\
$\eta_{b}$ & 9512.5 & 9398.0 & $\Upsilon$ & 9526.4 & 9460.30 \\
\hline
\end{tabular}

Note, our estimates represented in Tab. 1 are in reasonable agreement with the recent experimental data [2] with relative errors less than 1.8 per cent. 


\section{Leptonic decay constants}

The leptonic decay constants $f_{J}$ are important properties of mesons. In order to describe adequately the known 'sawtooth'-type unsmooth dependence of measured values of $f_{J}$ on meson masses (see Tab. 2), we have to introduce additional parameters $R_{J}$ characterizing the meson 'size' (in units of mass) into the meson ground-state functions as follows: $\tilde{U}_{R}(k)=$ $\int_{0}^{1} d s h(s) \exp \left[-s k^{2} / R_{J}^{2}\right]$, where $h(s)$ is a smooth function. Then, by keeping our model parameters fixed above and by varying on $R_{J}$ to fit recent data on $f_{J}$ we calculate optimal meson 'sizes' and the leptonic decay constants represented in Tab. 2.

Table 2. Estimated leptonic decay constants of mesons $f_{J}$ (in $\mathrm{MeV}$ ) compared to the experimental data

\begin{tabular}{llllllllll}
\hline $0^{-+}$ & $R_{f}$ & $f_{P}$ & Data & Ref. & $1^{--}$ & $R_{P}$ & $f_{V}$ & Data & Ref. \\
\hline$D$ & 0.93 & 207 & $206.7 \pm 8.9$ & {$[13]$} & $\rho$ & 0.33 & 221 & $221 \pm 1$ & {$[13]$} \\
$D_{s}$ & 1.08 & 257 & $257.5 \pm 6.1$ & {$[13]$} & $K^{*}$ & 0.38 & 217 & $217 \pm 7$ & {$[13]$} \\
$\eta_{c}$ & 1.83 & 238 & $238 \pm 8$ & {$[14]$} & $D^{*}$ & 0.78 & 245 & $245 \pm 20$ & {$[15]$} \\
$B$ & 1.73 & 193 & $192.8 \pm 9.9$ & {$[16]$} & $D_{s}^{*}$ & 0.90 & 271 & $272 \pm 26$ & {$[15]$} \\
$B_{s}$ & 2.18 & 239 & $238.8 \pm 9.5$ & {$[16]$} & $J / \Psi$ & 2.40 & 416 & $415 \pm 7$ & {$[13]$} \\
$B_{c}$ & 3.34 & 488 & $489 \pm 5$ & {$[14]$} & $B^{*}$ & 3.34 & 196 & $196 \pm 44$ & {$[15]$} \\
$\eta_{b}$ & 3.80 & 800 & $801 \pm 9$ & {$[14]$} & $\Upsilon$ & 2.80 & 715 & $715 \pm 5$ & {$[13]$} \\
\hline
\end{tabular}

\section{Dominant radiative decays of charmonium orbital excitations}

Nowadays, the charmonium states are intensively searched in different experiments [17].

The CCQM [4] represents an appropriate theoretical framework to perform the analysis of the recent measurement of the dominant (one-photon) radiative decays $\chi_{c J} \rightarrow J / \Psi+\gamma$ of the excited charmonium states $\chi_{c J},(J=0,1,2)$ reported by the LHCb Collaboration [18].

The invariant matrix element for the decay $\chi_{c J} \rightarrow \gamma+J / \Psi$ reads:

$$
\mathcal{M}\left(\chi_{c J}(p) \rightarrow J / \Psi\left(q_{1}\right)+\gamma\left(q_{2}\right)\right)=i(2 \pi)^{4} \delta^{(4)}\left(p-q_{1}-q_{2}\right) \varepsilon_{\chi}^{\Gamma} \varepsilon_{J / \Psi}^{\rho} \varepsilon_{\gamma}^{\sigma} T_{\Gamma \rho \sigma}\left(q_{1}, q_{2}\right),
$$

where $\left\{p, q_{1}, q_{2}\right\}$ and $\left\{\varepsilon_{\chi}^{\Gamma}, \varepsilon_{\gamma}^{\rho}, \varepsilon_{J / \Psi}^{\nu}\right\}$ are the momenta and polarization vectors of $\left\{\chi_{c J}, J / \Psi\right\}$ mesons and photon, correspondingly. In the leading order (LO), the matrix elements in Eq. (8) correspond to the Feynman diagrams of 'triangle' and 'bubble' types. Particularly, the LO amplitude given by the 'triangle' diagram reads:

$$
\begin{aligned}
T_{\Gamma \rho \sigma}\left(q_{1}, q_{2}\right) & =g_{\chi} g_{J / \Psi} e_{c} e N_{c} \int \frac{d^{4} k}{(2 \pi)^{4} i} \widetilde{\Phi}_{\chi}\left(-k^{2}\right) \widetilde{\Phi}_{J / \Psi}\left(-\left(k+\frac{1}{2} q_{2}\right)^{2}\right) \\
& \times \operatorname{tr}\left[\gamma_{\rho} S\left(\hat{k}+\frac{1}{2} \hat{p}\right) \Gamma_{\chi} S\left(\hat{k}-\frac{1}{2} \hat{p}\right) \gamma_{\sigma} S\left(\hat{k}-\frac{1}{2} \hat{p}+\hat{q}_{2}\right)\right],
\end{aligned}
$$

where $N_{c}=3, e_{c}$ and $e$ are the electric charge of c-quark and electron; $S(\hat{k})$ is the c-quark propagator, $\Gamma_{\chi}=\left\{I, \gamma_{\mu} \gamma_{5}, i\left(\gamma_{\mu} k_{v}+\gamma_{\nu} k_{\mu}\right)\right\}$ stand for the scalar, axial-vector and tensor currents in $\chi=\left\{\chi_{c 0}, \chi_{c 1}, \chi_{c 2}\right\}$ states.

The vertices $\widetilde{\Phi}_{\chi}\left(-k^{2}\right)$ and $\widetilde{\Phi}_{J / \Psi}\left(-k^{2}\right)$ are Gaussian functions and are determined by $\Lambda_{\chi_{c} J}$ and $\Lambda_{J / \Psi}$ - the corresponding 'size' parameters of $\chi_{c J}$ and $J / \Psi$ mesons.

The renormalized couplings $g_{\chi}$ and $g_{J / \Psi}$ for mesons $\chi=\left\{\chi_{c 0}, \chi_{c 1}, \chi_{c 2}\right\}$ and $J / \Psi$ are defined according the CCQM from the 'compositeness' condition by involving corresponding self-energy (mass) functions of mesons (see, for details [4]).

The 'bubble' diagrams take the similar structure but involving two $S$-propagators for the 'self-energy' quark-antiquark loop in the trace. 


\section{$\underline{\text { Spin }=0}$}

The invariant matrix element for the decay $\chi_{c 0} \rightarrow \gamma+J / \Psi$ of scalar charmonium takes the most general form with two independent four momenta (see, e.g. [19]):

$$
\mathcal{M}_{S V g} \sim \varepsilon_{\rho}\left(q_{1}\right) \cdot \varepsilon_{\sigma}\left(q_{2}\right) \cdot T_{\rho \sigma}, \quad T_{\rho \sigma}=d \cdot\left(q_{2}^{\rho} q_{1}^{\sigma}-g_{\rho \sigma}\left(q_{1} \cdot q_{2}\right)\right),
$$

where $T_{\rho \sigma}$ is the gauge invariant transition amplitude while form factor $d$ is determined by resolving Eq. (9) for $\Gamma_{J}=I$.

The decay width for the transition reads:

$$
\Gamma\left(\chi_{c 0} \rightarrow \gamma+J / \Psi\right)=\alpha / 24 \cdot M_{\chi_{c 0}}^{3}\left(1-M_{J / \Psi}^{2} / M_{\chi c 0}^{2}\right)^{3} \cdot g_{\chi c 0}^{2} \rightarrow \gamma+J / \Psi,
$$

where $\alpha=e^{2} / 4 \pi$ and the $\chi_{c 0}$-meson decay coupling $g_{\chi_{c 0} \rightarrow \gamma+J / \Psi}$ is calculated by solving Eq. (9).

\section{Spin=1}

For the axial-vector meson the invariant matrix element of transition $\chi_{c 1} \rightarrow \gamma+J / \Psi$ reads:

$$
\mathcal{M}_{A V g} \sim \varepsilon_{\mu}(p) \cdot \varepsilon_{\rho}\left(q_{1}\right) \cdot \varepsilon_{\sigma}\left(q_{2}\right) \cdot T_{\mu \rho \sigma},
$$

where the polarization vectors $\varepsilon_{\mu}, \varepsilon_{\rho}, \varepsilon_{\sigma}$ satisfy the constraints of transversality, completeness and orthonormality (see, e.g. [4]).

By taking into account the on-mass shell conditions one can write down five seemingly independent Lorentz structures of the gauge invariant transition amplitude as follows:

$$
\begin{aligned}
T_{\mu \rho \sigma}\left(q_{1}, q_{2}\right) & =\epsilon^{q_{2 \mu \sigma \rho}}\left(q_{1} \cdot q_{2}\right) W_{1}+\epsilon^{q_{1} q_{2} \sigma \rho} q_{1}^{\mu} W_{2} \\
& +\epsilon^{q_{1} q_{2} \mu \rho} q_{2}^{\sigma} W_{3}+\epsilon^{q_{1} q_{2} \mu \sigma} q_{1}^{\rho} W_{4}+\epsilon^{q_{1} \mu \sigma \rho}\left(q_{1} \cdot q_{2}\right) W_{5}
\end{aligned}
$$

with five independent terms. Further, by revealing specific symmetry relations between fourdimensional tensors $\epsilon^{\mu v \rho \sigma}$ one can reduce the number of independent Lorentz structures and rewrite down the gauge invariant transition amplitude as follows [20]:

$$
T_{\mu \rho \sigma}=\epsilon^{q_{1} q_{2} \mu \rho} q_{2}^{\sigma}\left(W_{1}+W_{3}-\frac{M_{J / \Psi}^{2}}{\left(q_{1} q_{2}\right)} W_{4}\right)+\epsilon^{q_{1} q_{2} \sigma \rho} q_{1}^{\mu}\left(W_{1}+W_{2}-\left(1+\frac{M_{J / \Psi}^{2}}{\left(q_{1} q_{2}\right)}\right) W_{4}\right),
$$

where form factors $W_{i}(i=1,2,3,4)$ are determined by evaluating Eq. (9) for $\Gamma_{\chi_{c 1}}=\gamma_{\mu} \gamma_{5}$.

The decay width of the one-photon radiative transition of axial-vector meson reads:

$$
\Gamma\left(\chi_{c 1} \rightarrow \gamma+J / \Psi\right)=1 /\left(12 \pi M_{\chi_{c 1}}^{2}\right)\left(\left|H_{L}\right|^{2}+\left|H_{T}\right|^{2}\right)\left(M_{\chi c 1}^{2}-M_{J / \Psi}^{2}\right) / 2 M_{\chi_{c 1}},
$$

where the helicity amplitudes $H_{L}$ and $H_{T}$ are expressed in terms of the Lorentz amplitudes $W_{i}$ and physical masses $M_{\chi_{c 1}}$ and $M_{J / \Psi}[20]$.

\section{Spin=2}

The invariant matrix element for the tensor meson radiative decay $\chi_{c 2} \rightarrow \gamma+J / \Psi$ reads:

$$
\mathcal{M}_{T V g} \sim \varepsilon_{\mu v}(p) \cdot \varepsilon_{\rho}\left(q_{1}\right) \cdot \varepsilon_{\sigma}\left(q_{2}\right) \cdot T_{\mu \nu \rho \sigma},
$$

where the polarization vectors $\varepsilon_{\mu v}, \varepsilon_{\rho}, \varepsilon_{\sigma}$ satisfy the transversality, tracelessness, completeness and orthonormality conditions. The Lorentz conditions for vector meson and photon, and the gauge invariance of the transition amplitude result in two independent Lorentz structures of the gauge invariant transition amplitude as follows:

$$
\begin{aligned}
T_{\mu \nu \rho \sigma} & =A \cdot\left(g^{\mu \rho}\left[g^{\sigma \nu}\left(q_{1} \cdot q_{2}\right)-q_{1}^{\sigma} q_{2}^{\nu}\right]+g^{\nu \rho}\left[g^{\sigma \mu}\left(q_{1} \cdot q_{2}\right)-q_{1}^{\sigma} q_{2}^{\mu}\right]\right) \\
& +B \cdot\left(g^{\sigma \rho}\left[q_{1}^{\mu} q_{2}^{v}+q_{1}^{v} q_{2}^{\mu}\right]-g^{\mu \sigma} q_{1}^{v} q_{2}^{\rho}-g^{\nu \sigma} q_{1}^{\mu} q_{2}^{\rho}\right)
\end{aligned}
$$


where two independent form factors $A$ and $B$ are determined by evaluating Eq. (9) for $\Gamma_{\chi_{c 2}}=$ $i\left(\gamma_{\mu} k_{v}+\gamma_{\nu} k_{\mu}\right)$. The decay width of the one-photon radiative transition of tensor meson reads:

$$
\Gamma\left(\chi_{c 2} \rightarrow \gamma+J / \Psi\right)=\alpha / 20 \cdot M_{\chi_{c 2}}^{3}\left(1-M_{J / \Psi}^{2} / M_{\chi_{c 2}^{2}}\right) \cdot g_{\chi_{c 2} \rightarrow \gamma+J / \Psi}^{2}
$$

where the decay coupling $g_{\chi_{c 2} \rightarrow \gamma+J / \Psi}$ of $\chi_{c 2}$ meson is calculated by resolving Eq. (9) in the CCQM approach.

The obtained results of estimations for the dominant one-photon radiative decay widths $\Gamma\left(\chi_{c J} \rightarrow J / \Psi+\gamma\right)$ of the charmonium orbital excitations for the CCQM model parameters $m_{c}=1.68 \mathrm{GeV}$ (the constituent mass of c-quark) and $\lambda=0.181 \mathrm{GeV}$ (the universal confinement scale) are represented in Tab. 3 in comparison with the recent experimental data [2].

Table 3. Estimated dominant one-photon radiative decay widths $\Gamma\left(\chi_{c J} \rightarrow J / \Psi+\gamma\right)$ (in units of $\mathrm{MeV}$ ) of the charmonium orbital excitations in comparison with the recent experimental data [2]

\begin{tabular}{c|c|c|c|c|c}
\hline Meson & Mass & $\Gamma_{\text {tot }}^{\text {expr }}(\mathrm{MeV})$ & $B R(\gamma+J / \Psi)$ & $\Gamma^{\text {expr }}$ & $\Gamma^{\text {theor }}$ \\
\hline$\chi_{c 0}\left(0^{++}\right)$ & $3414.71 \pm 0.30$ & $10.8 \pm 0.6$ & $(1.40 \pm 0.05) \%$ & $0.151 \pm 0.014$ & 0.150 \\
\hline$\chi_{c 1}\left(0^{++}\right)$ & $3510.67 \pm 0.05$ & $0.84 \pm 0.04$ & $(34.3 \pm 1.0) \%$ & $0.288 \pm 0.022$ & 0.289 \\
\hline$\chi_{c 2}\left(0^{++}\right)$ & $3556.17 \pm 0.07$ & $1.97 \pm 0.09$ & $(19.0 \pm 0.5) \%$ & $0.374 \pm 0.027$ & 0.373 \\
\hline
\end{tabular}

\section{References}

[1] S. Bethke, Eur. Phys. J. C 64, 689 (2009)

[2] M. Tanabashi et al. (Particle Data Group), Phys. Rev. D 98, 030001 (2018)

[3] G.V. Efimov and G. Ganbold, Phys. Rev. D 65, 054012 (2002). G. Ganbold, Phys. Part. Nuc., 43, 79 (2012). Phys. Part. Nuc. 45, 10 (2014)

[4] T. Branz, A. Faessler, T. Gutsche, M. A. Ivanov, J. G. Körner and V. E. Lyubovitskij, Phys. Rev. D 81, 034010 (2010). G. Ganbold, T. Gutsche, M. A. Ivanov and V. E. Lyubovitski, J. Phys. G: Nucl. Part. Phys. 42, 075002 (2015). M.A. Ivanov, J.G. Körner and C.T. Tran, Phys. Rev. D 94, 094028 (2016)

[5] G. Ganbold, Phys. Rev. D 81, 094008 (2010)

[6] G. Ganbold, Phys. Rev. D 79, 034034 (2009)

[7] C. S. Fischer, R. Alkofer and H. Reinhardt, Phys. Rev. D 65, 094008 (2002)

[8] S. J. Brodsky and de G. F. Teramond, Phys. Lett. B 582, 211 (2004)

[9] M. Baldicchi et al., Phys. Rev. D 77, 034013 (2008)

[10] C. J. Morningstar and M. Peardon, Phys. Rev. D 60, 034509 (1999)

[11] Y. Chen et al., Phys. Rev. D 73, 014516 (2006)

[12] E. Gregory et al., J. High Energ. Phys. 10, 170 (2012)

[13] K. A. Olive et al. (Particle Data Group Collaboration), Chin. Phys. C 38, 090001 (2014)

[14] T.-W. Chiu et al. (TWQCD Collaboration), Phys. Lett. B 651, 171 (2007)

[15] D. Becirevic et al., Phys. Rev. D 60, 074501 (1999)

[16] J. Laiho, E. Lunghi and R. S. van de Water, Phys. Rev. D 81, 034503 (2010)

[17] R. Aaij et al. (LHCb Collaboration), Phys. Rev. D 92, 112009 (2015). M. Ablikim et al. (BESIII Collaboration), Phys. Rev. Lett. 115, 221805 (2015). A. Zupanc et al. (Belle Collaboration), Phys. Rev. Lett. 113, 042002 (2014)

[18] R. Aaij et al. (LHCb Collaboration), Eur. Phys. J. C 77, 609 (2017)

[19] D. Gamermann, E. Oset and B. S. Zou, Eur. Phys. J. A 41, 85 (2009)

[20] S. Dubnicka, A.Z. Dubnickova, M.A. Ivanov, J.G. Körner, P. Santorelli and G.G. Saidullaeva, Phys. Rev. D 84, 014006 (2011) 